\title{
Raffination von Pflanzenölen für die Chemie durch Olefinmetathese
}

\author{
Samir Chikkali und Stefan Mecking*
}

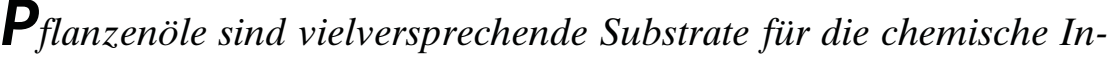
dustrie. Ihr Anwendungsbereich in der Produktion von Chemikalien kann durch moderne katalytische Verfahren erweitert werden. Ein illustratives Beispiel für die allgemeinen Erfordernisse der „Bioraffination" $z u$ Chemikalien ist die Olefinmetathese. Um diese Reaktion in industriellem Maßstab nutzen zu können, werden hoch aktive Katalysatoren benötigt, wodurch die Wahl der Metathesereaktion bestimmt wird. Die Mischung verschiedener Fettsäuren in technischen Pflanzenölen führt zu einer Vielzahl von Produkten. Diese Tatsache hat entscheidenden Einfluss auf das Verfahrensschema und kann zu neuen Chemikalien und Zwischenprodukten führen, die bisher noch nicht genutzt werden.
\end{abstract}

\section{Hintergrund}

Die chemische Industrie ist heutzutage größtenteils auf fossile Rohstoffe angewiesen. Nachwachsende Rohstoffe ${ }^{[1,2]}$ machen etwa ein Zehntel der verwendeten Ressourcen aus. Für eine Erhöhung dieses Anteils spricht eine Reihe von Gründen. In Anbetracht der begrenzten Verfügbarkeit von Erdöl und Erdgas werden langfristig Alternativen benötigt. Vermutlich werden Erdöl und in zunehmendem Maß auch Erdgas in den nächsten Jahrzehnten die wichtigsten Rohstoffe der chemischen Industrie bleiben, allein wegen der Tatsache, dass die Petrochemie bereits etabliert ist und Kohlenwasserstoffe wertvolle, energiereiche Ausgangsverbindungen zur Synthese von Chemikalien sind. Es ist aber anzunehmen, dass Alternativen wie Kohle oder nachwachsende Rohstoffe langsam an Bedeutung gewinnen werden. Kurzfristigere Gründe sind außer der Unabhängigkeit von den Erdöl fördernden Ländern auch eine geringere Abhängigkeit von schwankenden Rohölpreisen und die Umweltrisiken bei der Förderung von Erdöl aus der Tiefsee oder aus Ölsanden. Im Hinblick auf mögliche irreversible Auswirkungen auf das Klima ist eine Verringerung der Kohlendioxidemissionen wünschenswert. Heute ist allerdings allge-

[*] Dr. S. Chikkali, Prof. Dr. S. Mecking Universität Konstanz, Lehrstuhl für Chemische Materialwissenschaft, Fachbereich Chemie Universitätstraße 10, 78457 Konstanz (Deutschland) E-Mail: stefan.mecking@uni-konstanz.de mein anerkannt, dass nachwachsende Rohstoffe an sich keinen Vorteil in dieser Hinsicht bieten, sondern für jedes Verfahren und jedes Produkt eine $\mathrm{CO}_{2}$-Bilanz erstellt werden muss wenn auch für die meisten Herstellungsverfahren auf Kohlebasis angenommen werden kann, dass sie die schlechteste Option sind. Derartige Diskussionen setzen stets voraus, dass Wissenschaft und Technik überhaupt praktikable technische Verfahren beisteuern können.

Für die Synthese von Chemikalien sind Fettsäuren vielversprechende Substrate. ${ }^{[3-5]}$ Die Tatsache, dass Fettsäuren nur $2 \%$ der jährlich gebildeten Biomasse ausmachen, aber dennoch rund ein Drittel der von der chemischen Industrie eingesetzten nachwachsenden Rohstoffe fettsäurebasiert ist, unterstreicht dies. ${ }^{[5]}$ Etwa ein Sechstel der natürlichen Öle und Fette wird von der chemischen Industrie genutzt. ${ }^{[6]}$ Im Unterschied zu Kohlenhydraten und den meisten anderen Rohstoffen auf Biomassebasis sind Fettsäuren wegen ihrer langen Kohlenwasserstoffkette lipophil. Dies spiegelt sich in der Jahrtausende alten Anwendung ihrer Metallsalze als Seifen wider, in denen die Carboxylatgruppe als polare Kopfgruppe fungiert.

In vielen Fällen ist zusätzlich zur Carboxylatgruppe eine weitere Funktionalisierung der Fettsäure erwünscht. Hierfür bieten sich die Doppelbindungen ungesättigter Fettsäuren an. Ein bekanntes Beispiel ist die Epoxidierung der Triglyceride und weitere Umsetzung zu mehrfach funktionalisierten Vernetzern für Polyurethane. ${ }^{[7]}$ Bei diesen Anwendungen bleibt die Fettsäurekette intakt, es werden aber auch Reaktionen durchgeführt, die zur Spaltung der ungesättigten Fettsäure führen. Typischerweise wird dabei außer dem gewünschten difunktionellen Zielprodukt auch ein monofunktionelles Koppelprodukt erhalten. So werden durch Ozonolyse oder katalytische Oxidation der Doppelbindungen $\alpha, \omega$-Dicarbon- 
säuren mittlerer Kettenlänge und ihre Derivate zusammen mit monofunktionellen Oxidationsprodukten erhalten. Durch thermische Umlagerung kann aus Ricinolsäure Undecensäure erhalten werden, die weiter zu Nylon-11 umgesetzt wird. Zusätzlich fällt als Koppelprodukt Heptanal in stöchiometrischen Mengen an. Die Umsetzung von Ricinolsäure mit starken Basen bei erhöhter Temperatur führt zur CC-Bindungsspaltung, wobei 2-Octanol und Sebacinsäure erhalten werden; Sebacinsäure wird im Korrosionsschutz und zur Herstellung von Nylon-6,10 verwendet. Weitere Reaktionen, die in größerem Maßstab an Fettsäuren durchgeführt werden, sind Hydrierungen, Isomerisierungen, die fermentative $\omega$-Oxidation zu linearen Dicarbonsäuren und die Dimerisierung zu verzweigten Produkten. ${ }^{[3,5]}$ Dieses Repertoire kann möglicherweise durch katalytische C-C-Verknüpfungen ergänzt werden. Die Olefinmetathese ist ein hervorragendes Beispiel hierfür, das außerdem allgemeine Aspekte der Entwicklung neuer Konzepte zur industriellen Synthese von Chemikalien aus nachwachsenden Rohstoffen (durch rein chemische Syntheserouten) beleuchtet.

Umsetzungen von Fettsäuren und ihren Derivaten sind intensiv untersucht worden, ${ }^{[3,8]}$ und auch die Olefinmetathese solcher Substrate findet sowohl akademisches wie auch industrielles Interesse. ${ }^{[9]}$ Die Olefinmetathese wird unter anderem für die Synthese von biologisch aktiven Verbindungen und Feinchemikalien genutzt; hier können aus Pflanzenölen gewonnene Verbindungen eingesetzt werden. ${ }^{[10]}$ Dieser Kurzaufsatz behandelt dagegen Aspekte einer zukünftigen Erzeugung von Chemikalien aus Pflanzenölen durch Olefinmetathese im großen Maßstab.

\section{Metathesereaktionen ungesättigter Fettsäuren}

Die Olefinmetathese ${ }^{[11,12]}$ wird großtechnisch bereits seit fast 50 Jahren genutzt. Im Phillips-Verfahren wurde Propen in Ethen und Butene überführt (ein Verfahren, das heute umgekehrt durchgeführt wird) ${ }^{[13,14]}$ Bei diesem Verfahren werden auf Wolfram oder Rhenium basierende Heterogenkatalysatoren eingesetzt. Eine wesentliche Erweiterung des Potenzials der Metathese in der Synthese wurde mit dem Aufkommen definierter Metallalkylidenkomplexe als Katalysatorvorstufen erreicht. ${ }^{[15]}$ Das vielleicht größte Potenzial dieser
Katalysatoren ergibt sich aus ihrer Verträglichkeit mit funktionellen Gruppen.

Mit klassischen Heterogen- und In-situ-Katalysatoren können ungesättigte Fettsäuren und Öle umgesetzt werden. ${ }^{[16,17]}$ Es wird angenommen, dass die Leistung dieser Katalysatoren dabei durch den Grad ihrer Verträglichkeit mit funktionellen Gruppen begrenzt ist. ${ }^{[18]}$ Bei der Ethenolyse von Methyloleat wurden beispielsweise Umsatzzahlen von 500 erzielt.

Jüngere Entwicklungen umfassen die Selbstmetathese von Fettsäurederivaten ${ }^{[19]}$ sowie deren Kreuzmetathese mit funktionalisierten (z.B. Acrylaten, Acrylnitril oder Allylchlorid $^{[20,21]}$ ) sowie einfachen Olefinen wie Ethen. ${ }^{[22-29]}$ In diesen Untersuchungen wurde meist Methyloleat als Substrat verwendet. Besonders Rutheniumalkylidene, allen voran $\left[\left(\mathrm{PCy}_{3}\right)_{2} \mathrm{Cl}_{2} \mathrm{Ru}=\mathrm{CHPh}\right](\mathbf{1}$; Grubbs-Katalysator der 1. Generation, $\mathrm{Cy}=$ Cyclohexyl $)$ und $\left[\left(\mathrm{PCy}_{3}\right)(\eta-C\right.$ $\left.\left.\mathrm{C}_{3} \mathrm{H}_{4} \mathrm{~N}_{2} \mathrm{Mes}_{2}\right) \mathrm{Cl}_{2} \mathrm{Ru}=\mathrm{CHPh}\right] \quad(\mathbf{2} ;$ Grubbs-Katalysator der 2. Generation) wurden als Katalysatorvorstufen verwendet. Allerdings sind auch Alkylidenkomplexe anderer Metalle Vorstufen für sehr aktive Katalysatoren zur Metathese von Fettsäurederivaten. So werden z. B. mit Molybdänimidoalkylidenen ähnliche Produktivitäten beobachtet. ${ }^{[27]}$ Auch trägerfixierte, definierte Alkylidenspezies sind für diese Umsetzungen aktiv. ${ }^{[30]}$

Pflanzenöle enthalten in der Regel Fettsäuren unterschiedlicher Kettenlänge. Hinzu kommt, dass neben den einfach ungesättigten Verbindungen auch die mehrfach ungesättigten Analoga vorhanden sind (Tabelle 1). In diesem Zusammenhang sei angemerkt, dass die Zusammensetzung der Pflanzenöle mit der geographischen Herkunft sowie der Züchtung variieren kann. So können in ,high oleic“-Sonnenblumenöl bis zu 90 \% Ölsäure und in ,high erucic“-Rapsöl bis zu $50 \%$ Erucasäure enthalten sein. Eine Trennung ist zwar möglich, aber aufwändig, weshalb bei großtechnischen Verfahren die Zusammensetzung der eingesetzten Fettsäuren in der Regel die Zusammensetzung des genutzten Rohstoffs widerspiegeln wird. Dies ist ein Unterschied nicht nur zu vielen akademischen Untersuchungen der Metathese von Fettsäuren, sondern auch zu vielen homogen katalysierten industriellen Verfahren, in denen definierte (petrochemisch erzeugte) Ausgangsverbindungen eingesetzt werden. Die Metathese eines Gemisches aus Fettsäuren (oder ihren Es- 
Tabelle 1: Typische Zusammensetzung gängiger Pflanzenöle. ${ }^{[a][31,32]}$

\begin{tabular}{lllllll}
\hline Öl & $\begin{array}{l}\text { Doppel- } \\
\text { bindungen }^{[\mathrm{b}]}\end{array}$ & $\begin{array}{l}\text { Palmitat }^{[c]} \\
(16: 0)[\%]\end{array}$ & $\begin{array}{l}\text { Stearat } \\
(18: 0)[\%]\end{array}$ & $\begin{array}{l}\text { Oleat }^{[c]} \\
(18: 1)[\%]\end{array}$ & $\begin{array}{l}\text { Linoleat }^{[c]} \\
(18: 2)[\%]\end{array}$ & $\begin{array}{l}\text { Linolenat }^{[c]} \\
(18: 3)[\%]\end{array}$ \\
\hline Palmöl & 1.7 & 43 & 4 & 41 & 10 & - \\
Olivenöl & 2.8 & 14 & 3 & 72 & 10 & 0.6 \\
Rapsöl & 3.8 & 4 & 2 & 56 & 26 & 10 \\
Sojaöl & 4.6 & 11 & 4 & 23 & 53 & 8 \\
Sonnenblumenöl & 4.7 & 5 & 3 & 37 & 54 & 1 \\
HO-Sonnenblumenöl $^{[\mathrm{d}]}$ & 3.0 & 2.5 & 1.5 & 93 & 2.5 & - \\
\hline
\end{tabular}

[a] Gew.-\% der entsprechenden Fettsäure, bezogen auf den Gesamtanteil enthaltener Fettsäuren. Zusammensetzungen können je nach Herkunft variieren. [b] Durchschnittlicher Anteil an Doppelbindungen je Triglycerid. [c] Zahl der Kohlenstoffatome und Doppelbindungen in Klammern. [d] HO=high oleic.

tern) mit unterschiedlich vielen Doppelbindungen führt zu einer Vielzahl von Verbindungen, die mehr oder weniger gut getrennt werden können.

Pflanzenöle können zudem Verunreinigungen wie Wasser oder Peroxide enthalten, die zur Desaktivierung der Metathesekatalysatoren führen. Dies ist besonders bei industriellen Prozessen relevant, bei denen eine hohe Katalysatorproduktivität Voraussetzung ist (siehe Abschnitt 3). Allerdings verursacht das Entfernen der Verunreinigungen, z.B. durch Destillation oder Filtration über anorganische Adsorptionsmittel, ${ }^{[33]}$ zusätzliche Kosten.

\subsection{Selbstmetathese}

Die Selbstmetathese von Ölsäureestern führt zu linearen, einfach ungesättigten Kohlenwasserstoffen und $\alpha, \omega$-Diestern (Schema 1). Diese können durch Hydrierung zu den entsprechenden gesättigten Wachsen und langkettigen Diestern umgesetzt werden. Bei der Selbstmetathese von Methyloleat mit isolierten Rutheniumalkylidenen (z.B. 2) wurden mit Umsatzzahlen von mehreren Hunderttausend sehr hohe Katalysatorproduktivitäten beobachtet. ${ }^{[34]}$ Die vollständige Selbstmetathese liefert eine statistische 1:1:2-Mischung aus den beiden Produkten und dem Ausgangsmaterial (cis/transisomerisiert), es sei denn, ein Produkt kann selektiv aus dem Gleichgewicht entfernt werden. Ansonsten wären zur voll-

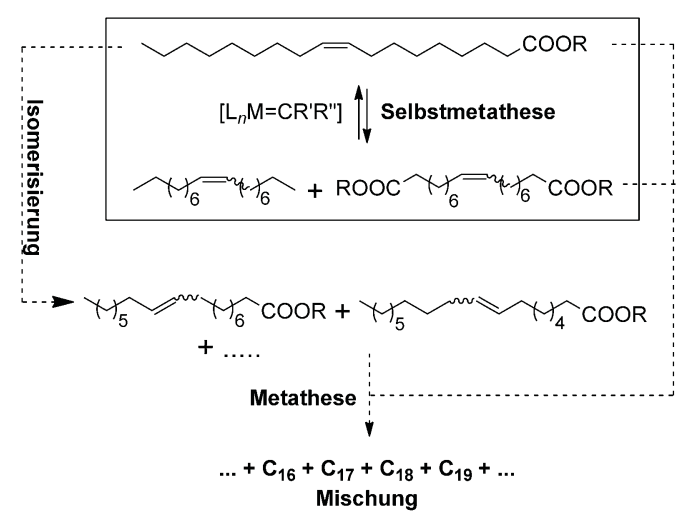

Schema 1. Selbstmetathese eines Fettsäureesters und Isomerisierung als mögliche Nebenreaktion. ständigen Umsetzung die Trennung des Reaktionsgemisches (z.B. durch Vakuumdestillation oder Kristallisation) und anschließende Rückführung der Ausgangsverbindung notwendig.

Eine mögliche Nebenreaktion ist die Isomerisierung von Doppelbindungen, ${ }^{[35]}$ die durch Zersetzungsprodukte des Metathesekatalysators gefördert wird. ${ }^{[36]}$ Diese Nebenreaktion wird hier am Beispiel der Selbstmetathese vorgestellt, kann aber auch bei anderen Metathesereaktionen auftreten. Die Kreuzmetathese der durch die Isomerisierung des Ausgangsmaterials und der während der primären Metathese gebildeten Produkte würde zu einer Reihe von einfach ungesättigten Kohlenwasserstoffen, $\alpha, \omega$-Diestern und Monoestern verschiedener Kettenlänge führen (Schema 1). Eine vollständige Trennung dieser Produkte wäre vermutlich nicht praktikabel.

Die Selbstmetathese von Triglyceriden (d.h. $R=$ trifunktionell) führt $\mathrm{zu}$ vernetzten, polymeren Strukturen. Diese können hydriert werden und finden Anwendung als Kerzenwachse und Bestandteile von Kosmetika. ${ }^{[37]}$

\subsection{Ethenolyse}

Durch Ethenolyse werden die Fettsäuren an der internen Doppelbindung gespalten und in endständige Olefine überführt (Schema 2, oben). Methyloleat wird so in 1-Decen und 9-Decensäuremethylester umgewandelt. Dies ist eine vielversprechende Reaktion, da endständige Olefine oft wertvolle Zwischenprodukte sind. Auch wenn die längerkettigen Produkte der Selbstmetathese (Schema 1) die gewünschten Produkte sind, könnte ein zusätzlicher Ethenolyseschritt von Vorteil sein, da die gebildeten Produkte (z.B. $\mathrm{C}_{10}$ für Oleat) wegen ihrer geringeren Molekulargewichte und Siedepunkte

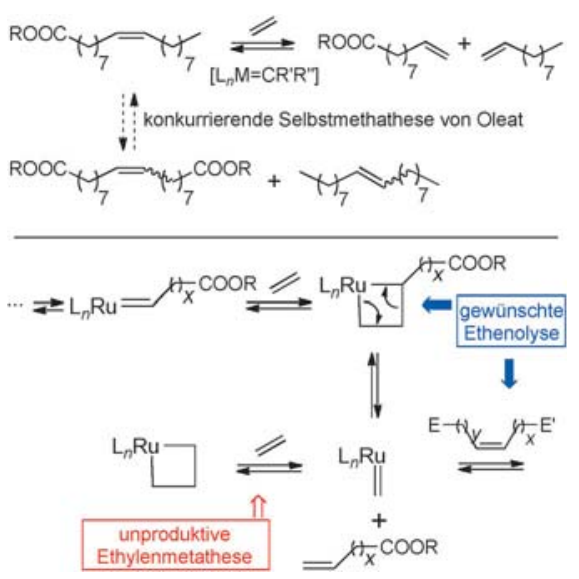

Schema 2. Ethenolyse eines Fettsäureesters (oben). Vereinfachtes Schema der unproduktiven Ethenmetathese (unten); E, E'=Alk(en)yl, Ester. 
unter Umständen besser aufgetrennt werden können. Im Anschluss an die Ethenolyse müsste die Selbstmetathese (unter Freisetzung von Ethen) der aufgereinigten 1-Olefine erfolgen. ${ }^{[38]}$ Dieses Vorgehen könnte besonders bei längerkettigen Substraten von Interesse sein.

Bei der Ethenolyse ist die Wahl der Reaktionsbedingungen ein Kompromiss zwischen Selektivität und Produktivität (bezogen auf den Substratumsatz). Eine hohe Ethenkonzentration erhöht die Selektivität für die Ethenolyse gegenüber der konkurrierenden Selbstmetathese des Substrats, gleichzeitig fördert sie aber auch die unproduktive Ethenmetathese (Schema 2, unten). Unsubstituierte Metallalkylidenkomplexe $\left(\left[\mathrm{L}_{n} \mathrm{Ru}=\mathrm{CH}_{2}\right]\right)$, die besonders bei Metathesereaktionen in Gegenwart von Ethen auftreten, haben eine höhere Neigung zur irreversiblen Desaktivierung ${ }^{[23,39-42]}$ als ihre substituierten Analoga $\left(\left[\mathrm{L}_{n} \mathrm{Ru}=\mathrm{CHR}\right]\right)$. Dies scheint ein entscheidender limitierender Faktor der Ethenolyse zu sein. Außer Desaktivierungsreaktionen, bei denen intrinsische Zwischenstufen des Katalysezyklus eine Rolle spielen, können auch Spuren von Wasser oder anderen polaren Molekülen (wie Alkoholen, Peroxiden oder Radikalbildnern, die durch die Substrate selbst eingebracht werden) zur Alkylidenzersetzung beitragen.

Über hohe Selektivitäten von $\geq 95 \%$ wurde für die Ethenolyse von Methyloleat an Rutheniumkatalysatoren mit $\mathrm{N}$-heterocyclischen Carbenliganden sowie an Molybdänalkylidenen berichtet. Die Produktivität beläuft sich im Allgemeinen auf Umsatzzahlen von mehreren Tausend (höhere Werte wurden bei niedrigen Umsätzen beobachtet). ${ }^{[23-27]}$ Dies liegt unter dem Wert von $5 \times 10^{4}$ Umsätzen je Metallzentrum, den verschiedene Firmen als notwendig ansehen, um einen Prozess im industriellen Maßstab wirtschaftlich zu betreiben. ${ }^{[23,24]}$

\subsection{Alkenolyse}

Im Unterschied zur Ethenolyse kann die Kreuzmetathese mit internen Olefinen, wie die Selbstmetathese in Abschnitt 2.1, mit sehr hohen Umsatzzahlen durchgeführt werden. Für die Kreuzmetathese von Methyloleat mit 2-Buten wurden Umsatzzahlen von bis zu $5 \times 10^{5}$ berichtet. ${ }^{[33,43]}$ Hierfür waren allerdings eine sorgfältige destillative Reinigung des Substrats sowie die Verwendung von reinem (Butadienfreiem) 2-Buten notwendig. Die bezüglich der Doppelbindung symmetrische Struktur von 2-Buten schränkt die Zahl der möglichen Reaktionsprodukte ein (unter der Voraussetzung, dass keine Isomerisierung stattfindet). Dennoch kann wegen der mehrfach ungesättigten Fettsäuren, die in technischen Pflanzenölen enthalten sind, eine Reihe von Produkten bei der Metathese gebildet werden. Dies ist in Schema 3 für die Butenolyse eines Triglycerids von $\mathrm{C}_{18}$-Fettsäuren dargestellt. Die Verwendung eines internen Olefins als Reaktant für die Alkenolyse verhindert die Bildung empfindlicher Rutheniummethylidenspezies. Es wurde gezeigt, dass die auftretenden Rutheniumethylidenspezies hochaktive Metathesekatalysatoren sind. ${ }^{[44]}$

Die Alkenolyse mit endständigen Olefinen kann ebenfalls mit relativ hohen Produktivitäten durchgeführt werden. Für
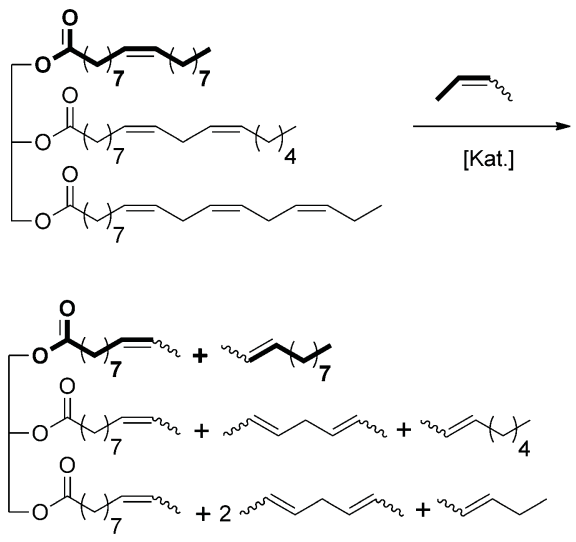

Schema 3. Butenolyse am Beispiel eines Triglycerids aus Ölsäure, Linolsäure und Linolensäure.

die Butenolyse mit 1-Buten wurden Umsatzzahlen von ca. $10^{5}$ berichtet. ${ }^{[33]}$ Dabei wird eine breitere Produktpalette als bei der Alkenolyse mit 2-Buten erhalten. Diese umfasst unter anderem die wertvollen 1-Olefine und intern sowie endständig ungesättigte Ester (Schema 4).

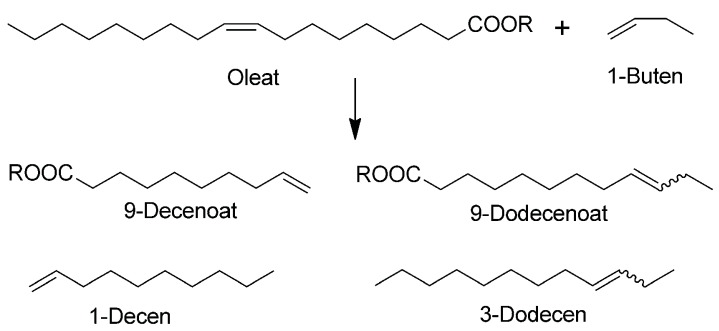

Schema 4. Hauptprodukte der Kreuzmetathese eines Oleats mit 1-Buten.

\section{Großtechnisches Szenario}

Bei einer Anwendung der Olefinmetathese von Pflanzenölen im industriellen Maßstab sind sowohl das gebildete Produktspektrum als auch die Katalysatorleistung entscheidende Kriterien. Aus den in Abschnitt 2 genannten Erwägungen kann die Alkenolyse mit einem 1-Olefin als Kompromiss zwischen diesen beiden Aspekten betrachtet werden. Im industriellen Maßstab sind kurzkettige Olefine wie Propen oder 1-Buten wegen ihrer niedrigen Kosten attraktive Substrate. Am Rande sei angemerkt, dass der Transport und die Lagerung außerhalb eines bestehenden Pipeline-Verbundes einfacher ist als im Fall von Ethen.

Für das eingesetzte Pflanzenöl ist ein hoher Gehalt an einfach ungesättigten Fettsäuren wünschenswert, wenn nicht die kurzkettigeren Verbindungen, die durch Metathese mehrfach ungesättigter Fettsäuren gebildet werden, als Produkte angestrebt werden. Unter den verfügbaren Pflanzenölen sticht Palmöl (Abbildung 1) mit Ölausbeuten von mehr als vier Tonnen je Hektar und Jahr deutlich gegenüber Sonnenblumen-, Raps- oder Sojaöl hervor. ${ }^{[45]}$ 


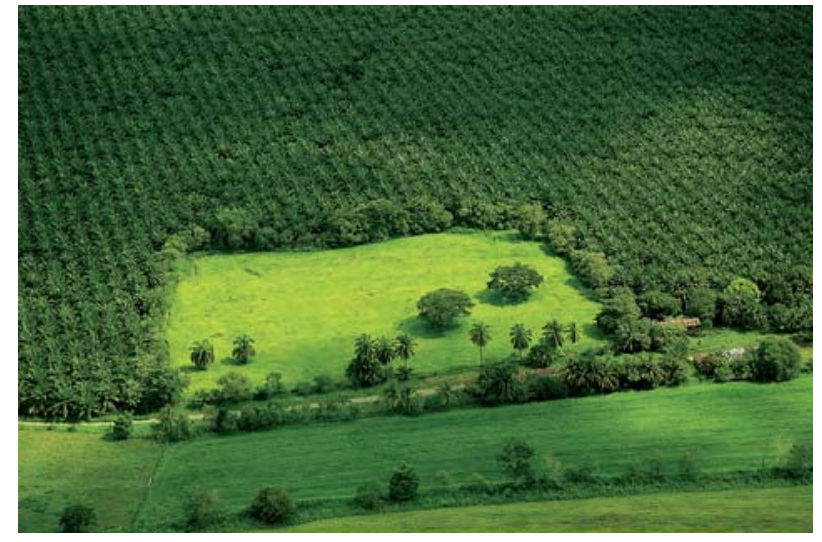

Abbildung 1. Palmenplantage.

Ein mögliches Verfahrensschema ${ }^{[46]}$ umfasst die Metathese von gereinigtem Pflanzenöl mit 1-Buten (Schemata 4 und 5). In diesem Schritt würde durch einen großen Überschuss von 1-Buten die Kreuzmetathese gegenüber der Selbstmetathese des Pflanzenöls begünstigt, aber auch die unerwünschte Selbstmetathese des 1-Butens gefördert. Daher werden die Reaktionsbedingungen vermutlich so gewählt werden, dass ein erheblicher Teil des Pflanzenöls der Selbstmetathese unterliegt. In einem Folgeschritt würden die Kohlenwasserstoffe (Olefine) von den Estern getrennt werden. Diese Trennung kann erleichtert werden, wenn die Metathese an den Triglyceriden durchgeführt wird, da die dabei entstehenden Ester wesentlich höhere Siedepunkte aufweisen als die Kohlenwasserstoffe. Nach dieser destillativen Trennung werden die Triglyceride umgeestert (Schema 5). Man beachte, dass gesättigte Fettsäuren, die in den Pflanzenölen enthalten sind (Tabelle 1), ebenfalls umgeestert werden (üblicherweise zu den Methylestern) und nach der Umesterung ebenso abgetrennt und isoliert werden (zur besseren Übersicht werden dieser Vorgang sowie die Abtrennung des Glycerins in Schema 5 nicht gezeigt). Außerdem ist zu bedenken, dass die Selbstmetathese von Triglyceriden, die in nicht un- erheblichem Maß als Nebenreaktion zur Butenolyse auftritt, zur Bildung von Oligomeren und Polymeren führt (siehe Abschnitt 2.1), was die Handhabung der Produktmischung wegen deren Viskosität erschweren kann.

Die auf diesem Weg aus Oleat gewonnenen 1-Olefine bestehen im Wesentlichen aus 1-Decen. Dessen Kettenlänge eignet sich gut, um z.B. durch Oligomerisierung Schmierstoffe zu erhalten. ${ }^{[47,48]}$ Interne Olefine, hauptsächlich 3-Dodecen, werden in vergleichbaren Mengen gebildet. Ein Beispiel für eine bestehende industrielle Nutzung von linearen, internen Olefinen in diesem C-Zahl-Bereich (wenn auch mit unterschiedlichen Positionen der Doppelbindung) ist die Umsetzung der Produkte $\left(\mathrm{C}_{11}\right.$ - bis $\mathrm{C}_{14}$-Olefine $)$ des auf Erdöl basierenden Shell-Higher-Olefinprozesses ${ }^{[49]} \mathrm{zu}$ linearen Alkylbenzolsulfonaten (LABS), die in Tensiden Anwendung finden. Die höhermolekulare Olefinfraktion aus der Selbstmetathese (mit $\mathrm{C}_{18}$ als Hauptkomponente) liefert nach der Hydrierung lineare Wachse. Die Hydrierung der gesamten Olefinfraktion liefert $n$-Alkane $\left(\mathrm{C}_{8}-\mathrm{C}_{18}\right)$, die als Bestandteile von Diesel oder Düsentreibstoff geeignet sind. ${ }^{[46]}$

Die Umesterung der Triglyceridfraktion liefert unter anderem einen $\omega$-ungesättigten Monoester. Ausgehend von Oleat wird 9-Decenoat gebildet. Solche Verbindungen sind antimikrobiell wirksam. ${ }^{[50,51]}$ In ähnlichem Umfang werden interne ungesättigte Ester, hauptsächlich 9-Dodecenoat, gebildet. Diese Ester können ebenfalls in antimikrobiellen Beschichtungen $^{[51]}$ oder als Zwischenprodukte für die Herstellung von Schmiermitteln ${ }^{[47 c]}$ oder Reinigungsmitteln verwendet werden. Die Doppelbindung kann nicht nur unter Bildung der gesättigten linearen Monoester hydriert, sondern auch in andere funktionelle Gruppen überführt werden. So können durch Carbonylierung, Hydrocyanierung oder Kreuzmetathese lineare $\alpha, \omega$-difunktionelle $\mathrm{C}_{11^{-}}$bis $\mathrm{C}_{14}$-Ester erhalten werden, die über eine Carboxylat- oder Nitrilfunktion verfügen, die zu einer Aminogruppe umgesetzt werden kann. ${ }^{[52]}$ $\omega$-Aminocarbonsäuren in diesem Kettenlängenbereich werden z. B. als AB-Monomere für die Herstellung von Polyamiden (Nylons) eingesetzt.

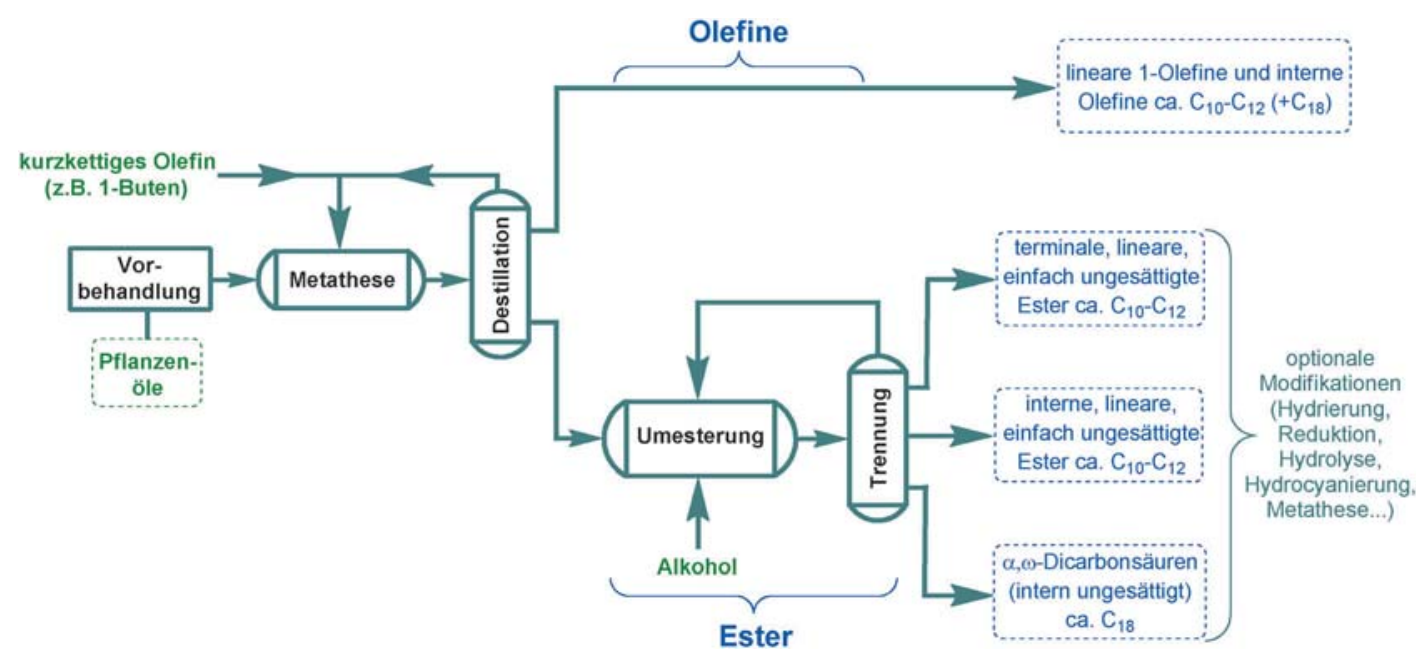

Schema 5. Schema eines Alkenolyseverfahrens zur Umsetzung der Triglyceride von Pflanzenölen zu mittel- bis langkettigen Olefinen und Estern. (Die angegebenen Kettenlängen der Produkte sind bezogen auf $\mathrm{C}_{18}$-Einsatzstoffe mit Oleat als Hauptkomponente. Produkte aus gesättigten Fettsäuren im Einsatzstoff sind nicht gezeigt.) 
Die in der Esterfraktion enthaltenen Produkte der Selbstmetathese liefern $\alpha, \omega$-funktionalisierte lineare Diester. Beispielsweise werden aus Oleaten nach der Hydrierung die Ester der 1,18-Octadecandicarbonsäure erhalten. Hierzu sei angemerkt, dass eine Synthese solcher Verbindungen durch $\omega$-Oxidation gesättigter Fettsäuren ebenfalls entwickelt wurde (insbesondere die Umwandlung von Stearinsäure in 1,18Octadecandicarbonsäure). ${ }^{[53]}$ Die Metathese bietet somit eine Alternative zu diesem biotechnologischen Verfahren. Solche langkettigen $\alpha, \omega$-difunktionellen Verbindungen sind von Interesse, da sie als (Co-)Monomere in Polykondensationsreaktionen zu Polyestern, Polyamiden oder Polyurethanen umgesetzt werden können. So ergibt die Kristallisation der langen Methylensequenzen bei langkettigen aliphatischen Polyestern Schmelzpunkte, die prinzipiell hoch genug für eine thermoplastische Verarbeitung sind. ${ }^{[54]}$ In Polyamiden wird durch die erhöhte Hydrophobie die Wasseraufnahme gegenüber jener von bestehenden Nylons verringert.

Sofern mehrfach ungesättigte Fettsäuren im eingesetzten Pflanzenöl in nennenswerten Mengen enthalten sind (Tabelle 1), führen sie zu zahlreichen weiteren Produkten. So ergibt die Metathese von Linoleat mit 1-Buten verschiedene einund zweifach ungesättigte $\mathrm{C}_{5^{-}}$bis $\mathrm{C}_{12}$-Kohlenwasserstoffe. Die Kreuzmetathese von Linoleat mit Oleat führt zur Bildung weiterer Kohlenwasserstoffe und Ester mit anderen Kettenlängen als denen der oben diskutierten $\mathrm{C}_{18}$-Verbindungen.

Es wurde auch vorgeschlagen, dass das Produktgemisch der Metathese ohne weitere Trennung als Treibstoff verwendet werden kann. Eine sich anschließende Reihe von katalytischen Hydrierungen, zum Abschluss bei hoher Temperatur, sättigt alle Doppelbindungen und führt dabei auch zur (teilweisen) Umsetzung der Ester zu Alkanen. Dadurch ergibt das Metatheseprodukt von Sojaöl mit 1-Buten eine Mischung, die überwiegend aus $\mathrm{C}_{9}$ - bis $\mathrm{C}_{18}$-Alkanen besteht und als Kerosinersatz vorgeschlagen wurde. ${ }^{[5]}$

In Bezug auf die mögliche Realisierung im industriellen Maßstab wurde von Elevance (einem Gemeinschaftsunternehmen der Firmen Materia und Cargill) der Bau einer Anlage angekündigt, in der Pflanzenöle mit 1-Buten umgesetzt werden sollen. ${ }^{[56,57]}$ Geplant wird eine Kapazität von zunächst 185000 Jahrestonnen. Die Anlage, in der Palmöl als Substrat eingesetzt werden werden soll (Abbildung 1), soll sich in der Nähe von Surabaya (Indonesien) im Bau befinden. Sie ist als Teil einer Bioraffinerie der Firma Wilmar International, einem weltweiten Verarbeiter von Palm-, Palmkern- und Kokosöl, konzipiert. Nach Angaben von Elevance wurde andernorts bereits ein Produktionslauf (vermutlich unter Einsatz derselben Technologie) im 500-Tonnen-Maßstab durchgeführt. ${ }^{[58]}$

\section{Schlussfolgerung und Bewertung}

Bestehende Anwendungen von Pflanzenölen als Rohstoffe für die Produktion von Chemikalien nutzen diese zur Erzeugung mittel- und langkettiger, ein- und zweifach funktionalisierter Kohlenwasserstoffe. Die Tatsache, dass es sich bei den Pflanzenölen um einen nachwachsenden Rohstoff handelt, ist im Prinzip nicht entscheidend. Gegenwärtig be- steht allerdings seitens der Kunden der chemischen Industrie eine wachsende Nachfrage nach Produkten, die aus nachwachsenden Rohstoffen hergestellt werden. Dies kann die Einführung neuer Verfahren und Produkte befördern - unter der Voraussetzung, dass deren Kosten und Nutzen nicht hinter bestehenden Technologien zurückstehen. Diese Erfordernis mag vorübergehend durch politische Regulierung eingeschränkt werden, wie das Beispiel von Kraftstoffen zeigt, in denen in vielen Ländern ein gewisser Anteil nachwachsender Rohstoffe enthalten sein muss. Auch sind höhere Preise bei manchen „life-style"-Nischenprodukten unter Umständen vertretbar. Um sich erfolgreich durchsetzen zu können, müssen die hier beschriebenen großtechnischen Verfahren jedoch auch auf längere Sicht wettbewerbsfähig sein. In diesem Zusammenhang wurde angegeben, dass die Kapital- wie auch die Betriebskosten für die Umwandlung von Pflanzenölen durch Metathese niedriger seien als bei vergleichbaren petrochemischen Verfahren. ${ }^{[57]}$

Was die Erzeugung des Rohstoffs angeht, so sind die Hektarerträge von Ölpflanzen gegenüber denen anderer Nutzpflanzen nicht besonders hoch. Allerdings liefern sie energiereiche funktionalisierte Kohlenwasserstoffe, die einen geeigneten Einstieg in die Synthese bestimmter Chemikalien bieten. Da Pflanzenöle keine besonders billigen Rohstoffe sind, erfordern neue Wege zu ihrer Umsetzung und Veredlung effiziente Verfahren, wie die hier vorgestellte Olefinmetathese veranschaulicht.

Hinsichtlich der viel diskutierten Konkurrenz mit der Erzeugung von Lebensmitteln ist die beispielhafte Betrachtung der Herstellung eines neuen Kunststoffes aus nachwachsenden Rohstoffen interessant. Realistischerweise würde ein solches Material allenfalls einen kleinen Teil der Gesamtproduktion von Kunststoffen ausmachen, was darin begründet ist, dass jedes solche Material ein sehr spezifisches Eigenschaftsprofil aufweist, das für eine gegebene Anwendung viele Voraussetzungen erfüllen muss. Selbst bei einem Massenkunststoff wie Polyethylen wird zwischen sehr vielen verschiedenen Typen unterschieden. Die weltweite Produktion von Biodiesel beträgt momentan etwa 20 Millionen Tonnen pro Jahr, was aber nur etwa $1 \%$ des jährlichen Kraftstoffverbrauchs entspricht. Dagegen ist es eine anspruchsvolle und kostenintensive Aufgabe, 100000 Tonnen eines neuen Polymers oder einer neuen Chemikalie auf den Markt zu bringen. Natürlich gilt dieses Argument nur in beschränktem Maß für bekannte Monomere, die aus nachwachsenden Rohstoffen hergestellt werden, z. B. Ethen aus Zuckerrohr oder 1-Olefine aus Pflanzenöl. Aus einem anderen Blickwinkel betrachtet, wäre die Annahme, dass $10 \%$ der jährlichen Polymerproduktion von 260 Millionen Tonnen mittelfristig durch neue Polymere aus nachwachsenden Rohstoffen ersetzt werden würden, eher optimistisch. Dies zeigt wiederum, dass die Konkurrenz mit der Nahrungsmittelproduktion, besonders bei der Herstellung von technischen Kunststoffen, Spezial- und Feinchemikalien, unter rationalen Gesichtspunkten eher unproblematisch ist.

Die Metathese technischer Pflanzenöle führt wegen der mehrfach ungesättigten Fettsäuren zwangsläufig zu einer ganzen Reihe von Kohlenwasserstoffen und Estern als Produkten. Typischerweise führen Metathesereaktionen $\mathrm{zu}$ 
Gleichgewichten der Reaktanten, ohne eine starke kinetische Bevorzugung eines bestimmten Produktes. Daher werden Mischungen verschiedener Produkte gebildet, besonders wenn unsymmetrische Olefine (z.B. 1-Buten im obigen Beispiel) eingesetzt werden. Ein grundlegender Vorteil der Metathese ist die Bildung ausschließlich linearer Produkte. Der Begriff „Bioraffinerie“, ${ }^{[59]}$ der oft sehr breit verwendet wird, beschreibt in diesem Fall recht gut die Umwandlung eines Rohstoffs in eine größere Zahl von Produkten, wie dies auch in einer petrochemischen Raffinerie der Fall ist. Im Unterschied zur herkömmlichen petrochemischen Raffinerie, bei der Basischemikalien wie Ethen oder Propen erhalten werden, die als Ausgangsstoffe für die Synthese von zahlreichen Intermediaten eingesetzt werden, entsprechen die hier erhaltenen Produkte aber eher heutigen Zwischenprodukten. Für einige dieser Verbindungen besteht ein klarer Bedarf, da sie bereits als Zwischenprodukte verwendet werden. Andere Produkte der betrachteten Pflanzenölmetathese hingegen erfordern neue Anwendungen und Absatzmärkte. Die Entwicklung und die Ökonomie dieser neuen Anwendungen sowie die Wirtschaftlichkeit der Verfahren einschließlich der Leistung der Metathesekatalysatoren werden entscheidend für den Erfolg sein.

Da Untersuchungen im Labor- und Pilotmaßstab kein vollständiges Bild der tatsächlichen praktischen Durchführbarkeit in großen Anlagen ergeben können, werden die zukünftigen Entwicklungen auf diesem Gebiet wertvolle Erkenntnisse zur Praktikabilität der Olefinmetathese in industriellen Verfahren liefern. Ebenso könnten neue Wege zur Umwandlung von Pflanzenölen in Chemikalien aufgezeigt werden, wie auch die potenziell verfügbaren neuen Zwischenstufen zur Entwicklung neuer Spezialchemikalien und Polymere inspirieren.

S.C. dankt der Alexander von Humboldt-Stiftung für ein Forschungsstipendium. Wir danken der Stiftung Baden Württemberg (Programm Umwelttechnologieforschung) für die finanzielle Unterstützung unserer Untersuchungen zu nachwachsenden Rohstoffen.

[1] D. R. Dodds, R. A. Gross, Science 2007, 318, 1250-1251.

[2] S. Mecking, Angew. Chem. 2004, 116, 1096-1104; Angew. Chem. Int. Ed. 2004, 43, 1078-1085.

[3] U. Biermann, U. Bornscheuer, M. A. R. Meier, J. O. Metzger, H. J. Schäfer, Angew. Chem. 2011, 123, 3938-3956; Angew. Chem. Int. Ed. 2011, 50, 3854-3871.

[4] Y. Xia, R. C. Larock, Green Chem. 2010, 12, 1893-1909.

[5] „Fatty Acids“: D. J. Anneken, S. Both, R. Christoph, G. Fieg, U. Steinberner, A. Westfechtel in Ullmann's Encyclopedia of Industrial Chemistry, 6. Aufl. (Hrsg.: W. Gerhartz, B. Elvers), Wiley-VCH, Weinheim, 2006.

[6] Die Gesamtproduktion liegt bei ca. 125 Millionen Tonnen pro Jahr. Darin enthalten sind auch tierische Fette, die allerdings nur eine untergeordnete Rolle (20\%) gegenüber Pflanzenölen $(80 \%)$ spielen.

[7] Z. S. Petrovic, Polym. Rev. 2008, 48, 109-155.
[8] A. Behr, J. Perez-Gomez, Eur. J. Lipid Sci. Technol. 2010, 112, $31-50$.

[9] P. Malacea, P. H. Dixneuf in Green Metathesis Chemistry (Hrsg.: V. Dragutan, A. Demonceau, I. Dragutan), Springer, Berlin, 2010, S. 185-206 (NATO Science for Peace and Security Series-A: Chemistry and Biology).

[10] D. Bicchielli, Y. Borguet, L. Delaude, A. Demonceau, I. Dragutan, V. Dragutan, C. Jossifov, R. Kalinova, F. Nicks, X. Sauvage in Green Metathesis Chemistry (Hrsg.: V. Dragutan, A. Demonceau, I. Dragutan), Springer, Berlin, 2010, S. 207-274 (NATO Science for Peace and Security Series-A: Chemistry and Biology).

[11] N. Calderon, H. Y. Chen, K. W. Scott, Tetrahedron Lett. 1967, 8, $3327-3329$.

[12] J. L. Hérisson, Y. Chauvin, Makromol. Chem. 1971, 141, 161 167.

[13] J. C. Mol, J. Mol. Catal. A 2004, 213, 39-45.

[14] a) A. Scott, Chem. Week 1999, 161, 3. November, 41; b) N. Alperowicz, Chem. Week 2002, 164, 6. März, 16.

[15] a) R. R. Schrock, Angew. Chem. 2006, 118, 3832-3844; Angew. Chem. Int. Ed. 2006, 45, 3748-3759; b) R. H. Grubbs, Angew. Chem. 2006, 118, 3845-3850; Angew. Chem. Int. Ed. 2006, 45, $3760-3765$.

[16] a) P. B. Van Dam, M. C. Mittelmeijer, C. Boelhouwer, J. Chem. Soc. Chem. Commun. 1972, 1221 -1222; b) P. B. Van Dam, M. C. Mittelmeijer, C. Boelhouwer, J. Am. Oil Chem. Soc. 1974, 51, 389-392; c) E. Verkuijlen, F. Kapteijn, J. C. Mol, C. Boelhouwer, J. Chem. Soc. Chem. Commun. 1977, 198-199; d) C. Boelhouwer, J. C. Mol, Prog. Lipid Res. 1985, 24, 243-267.

[17] J. C. Mol, Green Chem. 2002, 4, 5-13.

[18] J. C. Mol, J. Mol. Catal. 1994, 90, 185-199.

[19] H. L. Ngo, K. Jones, T. A. Foglia, J. Am. Oil Chem. Soc. 2006, 83, $629-634$.

[20] a) X. Miao, A. Blokhin, A. Pasynskii, S. Nefedov, S. N. Osipov, T. Roisnel, C. Bruneau, P. H. Dixneuf, Organometallics 2010, 29, 5257-5262; b) R. Malacea, C. Fischmeister, C. Bruneau, J. L. Dubois, J. L. Couturier, P. H. Dixneuf, Green Chem. 2009, 11, $152-155$; c) X. Miao, P. H. Dixneuf, C. Fischmeister, C. Bruneau, Green Chem. 2011, 13, 2258-2271.

[21] a) A. Rybak, M. A. R. Meier, Green Chem. 2007, 9, 1356-1361; b) T. Jacobs, A. Rybak, M. A. R. Meier, Appl. Catal. A 2009, 353, $32-35$.

[22] S. Warwel, F. Bruse, C. Demes, M. Kunz, M. R. G. Klaas, Chemosphere 2001, 43, 39-48.

[23] K. A. Burdett, L. D. Harris, P. Margl, B. R. Maughon, T. Mokhtar-Zadeh, P. C. Saucier, E. P. Wasserman, Organometallics 2004, 23, 2027-2047.

[24] Y. Schrodi, T. Ung, A. Vargas, G. Mkrtumyan, C. W. Lee, T. M. Champagne, R. L. Pederson, S. K. Hong, Clean Soil Air Water 2008, 36, 669-673.

[25] D. R. Anderson, T. Ung, G. Mkrtumyan, G. Bertrand, R. H. Grubbs, Y. Schrodi, Organometallics 2008, 27, 563-566.

[26] R. M. Thomas, B. J. Keitz, T. M. Champagne, R. H. Grubbs, J. Am. Chem. Soc. 2011, 133, 7490-7496.

[27] S. C. Marinescu, R. R. Schrock, P. Müller, A. H. Hoveyda, J. Am. Chem. Soc. 2009, 131, 10840-10841.

[28] C. Thurier, C. Fischmeister, C. Bruneau, H. Olivier-Bourbigou, P. H. Dixneuf, ChemSusChem 2008, 1, 118-122.

[29] R. E. Montenegro, M. A. R. Meier, Eur. J. Lipid Sci. Technol. 2012, 114, 55-62.

[30] a) F. Blanc, J. Thivolle-Cazat, J.-M. Basset, C. Coperet, A. S. Hock, Z. J. Tonzetich, R. R. Schrock, J. Am. Chem. Soc. 2007, 129, 1044-1045; b) I. Karamé, M. Boualleg, J.-M. Camus, T. K. Maishal, J. Alauzun, J.-M. Basset, C. Coperet, R. J. P. Corriu, E. Jeanneau, A. Mehdi, C. Rey, L. Veyre, C. Thieuleux, Chem. Eur. J. 2009, 15, 11820-11823. 
[31] M. N. Belgacem, A. Gandini in Monomers, Polymers and Composites from Renewable Resources (Hrsg.: M. N. Belgacem, A. Gandini), Elsevier, Amsterdam, 2008, S. 39-66.

[32] „Fats and Fatty Oils“: A. Thomas in Ullmann's Encyclopedia of Industrial Chemistry, 6. Aufl. (Hrsg.: W. Gerhartz, B. Elvers), Wiley-VCH, Weinheim, 2005.

[33] Y. Schrodi, R. L. Pederson, H. Kaido, M. J. Tupy (Materia Inc.), WO 2008/046106A2, 2008.

[34] M. B. Dinger, J. C. Mol, Adv. Synth. Catal. 2002, 344, 671-677.

[35] a) F. C. Courchay, J. C. Sworen, K. B. Wagener, Macromolecules 2003, 36, 8231-8239; b) G. B. Djigoué, M. A. R. Meier, Appl. Catal. A 2009, 368, 158-162.

[36] a) S. H. Hong, M. W. Day, R. H. Grubbs, J. Am. Chem. Soc. 2004, 126, 7414-7415; b) S. H. Hong, D. P. Sanders, C. W. Lee, R. H. Grubbs, J. Am. Chem. Soc. 2005, 127, 17160-17161; c) B. Schmidt, J. Mol. Catal. 2006, 254, 53-57.

[37] K. D. Uptain, T. A. Murphy, S. Walters (Elevance Renewable Sciences Inc.), WO 2008/151064A1, 2008.

[38] S. Warwel, C. Demes, G. Steinke, J. Polym. Sci. Part A 2001, 39 , $1601-1609$.

[39] M. Ulman, R. H. Grubbs, J. Org. Chem. 1999, 64, 7202-7207.

[40] W. J. van Rensburg, P. J. Steynberg, W. H. Meyer, M. M. Kirk, G. S. Forman, J. Am. Chem. Soc. 2004, 126, 14332-14333.

[41] S. H. Hong, A. G. Wenzel, T. T. Salguero, M. W. Day, R. H. Grubbs, J. Am. Chem. Soc. 2007, 129, 7961-7968.

[42] K. Vehlow, S. Gessler, S. Blechert, Angew. Chem. 2007, 119, 8228-8231; Angew. Chem. Int. Ed. 2007, 46, 8082-8085.

[43] a) J. Patel, J. Elaridi, W. R. Jackson, A. J. Robinson, A. K. Serelis, C. Such, Chem. Commun. 2005, 5546-5547; b) J. Patel, S. Mujcinovic, W. R. Jackson, A. J. Robinson, A. K. Serelis, C. Such, Green Chem. 2006, 8, 450-454.

[44] a) M. S. Sanford, J. A. Love, R. H. Grubbs, J. Am. Chem. Soc. 2001, 123, 6543-6554; b) S. E. Lehman, K. B. Wagener, Organometallics 2005, 24, 1477-1482.

[45] a) Y. Basiron, Eur. J. Lipid Sci. Technol. 2007, 109, 289-295; b) S. Mekhilef, S. Siga, R. Saidur, Renewable Sustainable Energy Rev. 2011, 15, 1937-1949.

[46] S. A. Cohen, M. L. Luetkens, C. Balakrishnan, R. Snyder (Elevance Renewable Sciences), WO 2011/046872A2, 2011.
[47] a) S. Boyde, Green Chem. 2002, 4, 293-307; b) G. D. Yadav, N. S. Doshi, Green Chem. 2002, 4, 528-540; c) „Lubricants and Lubrication": T. Bartels, W. Bock, J. Braun, C. Busch, W. Buss, W. Dresel, C. Freiler, R.-P. Heckler, D. Hörner, F. Kubicki, G. Lingg, A. Losch, R. Luther, T. Mang, S. Noll, J. Omeis in Ullmann's Encyclopedia of Industrial Chemistry, 6. Aufl. (Hrsg.: W. Gerhartz, B. Elvers), Wiley-VCH, Weinheim, 2005.

[48] M. M.-S. Wu, K. S. Colle, R. Y. Saleh, A. D. Godwin, J. E. R. Stannat (ExxonMobil), US 2010/0160506A1, 2010.

[49] „Organic-organic biphasic catalysis“: D. Vogt in Multiphase Homogeneous Catalysis (Hrsg.: B. Cornils, W. A. Herrmann, I. T. Horvath, W. Leitner, S. Mecking, H. Olivier-Bourbigou, D. Vogt), Wiley-VCH, Weinheim, 2005, S. 330-335.

[50] a) R. G. Binder, B. G. Chan, C. A. Elliger, Agric. Biol. Chem. 1979, 43, 2467-2471; b) M. Nakatani, Y. Fukunaga, H. Haraguchi, M. Taniguchi, Bull. Chem. Soc. Jpn. 1986, 59, 3535-3539.

[51] J. L. Zullo, J. C. Anderson, H. Kaido, R. L. Pederson, Y. Schrodi, W. H. Sperber, M. J. Tupy, E. H. Wagener, US 7951232B2, 2011.

[52] T. W. Abraham, H. Kaido, C. W. Lee, R. L. Pederson, Y. Schrodi, M. J. Tupy (Cargill and Materia Inc.), WO 2008/048520A2, 2008.

[53] a) S. Picataggio, T. Rohrer, K. Deanda, D. Lanning, R. Reynolds, J. Mielenz, L. D. Eirich, Nat. Biotechnol. 1992, 10, 894-898; b) U. Schörken, P. Kempers, Eur. J. Lipid Sci. Technol. 2009, 111, $627-645$.

[54] a) D. Quinzler, S. Mecking, Angew. Chem. 2010, 122, $4402-$ 4404; Angew. Chem. Int. Ed. 2010, 49, 4306-4308; b) F. Stempfle, D. Quinzler, I. Heckler, S. Mecking, Macromolecules 2011, 44, 4159-4166; c) J. Trzaskowski, D. Quinzler, C. Bährle, S. Mecking, Macromol. Rapid Commun. 2011, 32, 1352-1356.

[55] M. L. Luetkens, S. A. Cohen, C. Balakrishnan (Elevance Renewable Sciences), WO 2010/062958A1, 2010.

[56] Pressemitteilung „Elevance Renewable Sciences“ vom 28. Juni 2010.

[57] Angaben („Form S-1“) zum geplanten Börsengang von „Elevance Renewable Sciences“, bei der US-Börsenaufsichtsbehörde (SEC) am 20. September 2011 eingereicht.

[58] Pressemitteilung „Elevance Renewable Sciences“ vom 18. Mai 2011.

[59] B. Kamm, P. R. Gruber, M. Kamm, Biorefinieries-Industrial Processes and Products, Wiley-VCH, Weinheim, 2006. 